\title{
All-Metallic $\varepsilon$-Near-Zero (ENZ) Lens Based On Ultra-Narrow Hollow Rectangular Waveguides: Experimental Results
}

\author{
B. Orazbayev, V. Torres, V. Pacheco- \\ Peña, F. Falcone, J. Teniente, M. \\ Beruete, M.Sorolla \\ Dept. of Electrical and Electronic \\ Engineering \\ Antennas Group - TERALAB, \\ Universidad Pública de \\ Navarra \\ Pamplona, Spain \\ b.orazbayev@unavarra.es \\ victor.torres@unavarra.es \\ victor.pacheco@unavarra.es \\ francisco.falcone@unavarra.es \\ jorge.teniente@unavarra.es \\ miguel.beruete@unavarra.es
}

\author{
M. Navarro-Cía \\ Dept. of Electrical and Electronic \\ Engineering Optical and Semiconductor \\ Devices Group, Imperial College \\ London \\ London SW7 2BT, United Kingdom \\ Centre for Plasmonics and \\ Metamaterials, Imperial College \\ London \\ London SW7 2AZ, United Kingdom \\ Centre for Terahertz Science and \\ Engineering, Imperial College London \\ London SW7 2AZ, United Kingdom \\ m.navarro@imperial.ac.uk
}

\author{
N. Engheta \\ Dept. Electrical and Systems \\ Engineering, University of \\ Pennsylvania, 200 South 33rd Street, \\ ESE 203 \\ Moore, Philadelphia, Pennsylvania \\ 19104, USA \\ engheta@ee.upenn.edu
}

\begin{abstract}
Here we perform numerical and experimental investigation of plano-concave all-metallic \&-near-zero (ENZ) lens with operational frequency $f=144 \mathrm{GHz}$. The ENZ lens is achieved by stacking an array of narrow hollow rectangular waveguides working near cut-off frequency. Focusing and radiation properties are numerically analyzed and measured. The enhancement of $5.61 \mathrm{~dB}$ and directivity of $17.6 \mathrm{dBi}$ are shown. Good agreement between experimental and numerical results is demonstrated.
\end{abstract}

Keywords—metamaterial lenses; ENZ media; millimeter waves

\section{INTRODUCTION}

For many years control of the transmission of light has been an important challenge. For instance, conventional dielectric lenses have been used to collimate light for centuries. However, due to the limited range of permittivity and permeability of natural materials, these lenses are often bulky and have limited range of applications. In recent years, appearance of metamaterials produced significant shift in the solution of this problem. Metamaterials, whose values of permittivity $(\varepsilon)$ or permeability $(\mu)$ can be designed at will, have been proposed to many applications, such as negative refraction, lenses, cloaking devices or even computational metamaterials [1]-[6].

More recently, metamaterials with an effective permittivity close to zero have gained interest, since they offer new exciting possibilities, such as supercoupling or energy squeezing [7]. Such ENZ materials make possible, under certain conditions (simultaneous relative permeability close to zero or crosssectional area electrically very small), to match bulk metamaterial impedance to free space and also provide small phase advance over a physically long distance for propagating electromagnetic waves in these media [7]. Based on these properties several different applications have been proposed [8]-[12].

A practical implementation of ENZ metamaterials is based on stacking perfectly electric conducting (PEC) metallic waveguides working at frequency close to cut-off frequency. In this communication, using such implementation, all-metallic ENZ lens is designed, fabricated and measured at frequency $144 \mathrm{GHz}$ [13]. Along with the transmission properties, the radiation characteristics are analyzed.

\section{LENS DESIGN}

The ENZ plano-concave lens is realized by employing a two-dimensional array of narrow hollow rectangular waveguides of dimensions $h_{x}$ and $h_{y}$, along $x$ and $y$ axes, where $h_{y} \ll h_{x}$. The aperture dimension perpendicular to the electric field excitation is $h_{x}=1.1 \pm 0.025 \mathrm{~mm}$ with the aim to have the cutoff frequency of the fundamental mode $\mathrm{TE}_{10}$ at $136.36 \mathrm{GHz}$. In order to have energy squeezing or bulk material impedance matched with free space, the dimension $h_{y}$ must be electrically very small [14], so it is designed with a value $h_{y}=0.05 \pm$ $0.02 \mathrm{~mm} \approx \lambda_{0} / 42$. The final design of whole ENZ lens consists of an array of $33 \times 144$ narrow hollow rectangular waveguides along $x$ and $y$ axes, respectively, with total dimensions $d_{x}=$ $46.2 \mathrm{~mm}, d_{y}=55.5 \mathrm{~mm}$ and $L_{z}=40 \mathrm{~mm}$. The total size of the lens excluding top and bottom bases is $L_{x}=76.2 \mathrm{~mm}, L_{y}=86.2$ $\mathrm{mm}$ and $L_{z}=40 \mathrm{~mm}$. Fabricated prototype is presented in Fig. 1. Due to the small phase variation in ENZ media, the phase front is conformal to the exit surface [15], [16]. Thus, the output face should have concave profile in order to focus an incoming plane wave. Following these principles, the ENZ lens is designed and fabricated with a flat face at input and a 
concave profiled face with diameter $d_{y}=55.5 \mathrm{~mm}$ at the output.

\section{RESULTS}

\section{A. Focusing properties}

The performance of ENZ lens was experimentally characterized by means of an Agilent Technologies N5242A PNA-X network analyzer. A scheme of experimental setup is shown in Fig. 2(a). A D-band corrugated horn antenna generating a $y$-polarized Gaussian beam was used as a source, placed $160 \mathrm{~mm}$ away from the flat face of the lens. A waveguide probe WR-8.0 was used as a receiver, which was fixed on a motorized 2-axis translation stage. Raster scanning was done with a minimum step of $0.8 \mathrm{~mm}$ in both $x$ and $y$ axes in a rectangle of $320 \mathrm{~mm} \times 320 \mathrm{~mm}$ which was $100 \mathrm{~mm}$ away from output of the lens. Both transmitter and receiver were connected to the Agilent VNA. The setup was covered with millimeter-wave absorbers with aim to minimize undesired reflections and scattering. A spatial field distribution in any $x y$ plane can be obtained from the measured components of the electric field by plane-to-plane translation, performed with fast Fourier transformation [17].

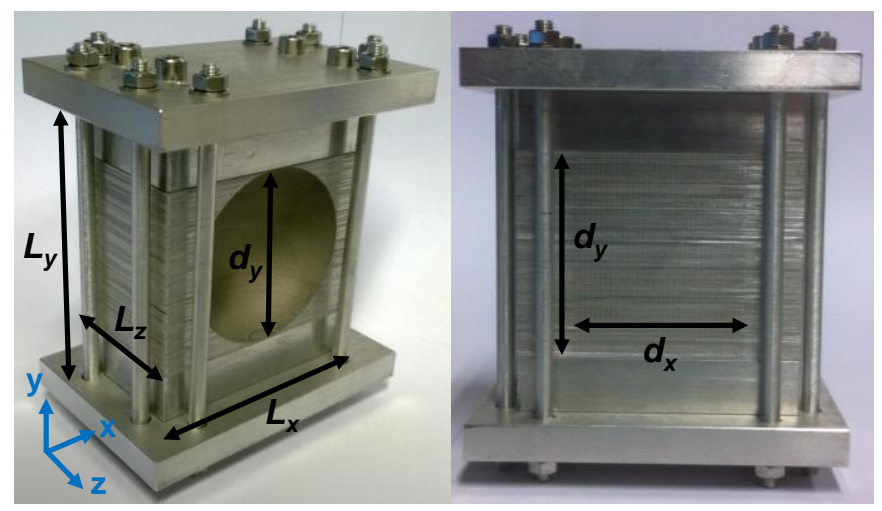

Fig. 1. Photograph of the plano-concave ENZ metallic lens.

First, in order to obtain the operational frequency of ENZ lens the experimental transmission enhancement spectra along the optical $z$-axis were obtained (Fig. 2(b) right inset). As it can be observed in this figure the maximum transmission occurs at $144 \mathrm{GHz}$. At this frequency effective permittivity $\varepsilon_{\text {eff }}=0.103$ is close to zero, accomplishing the condition of ENZ media and therefore it will be considered as working frequency. The focal length at this frequency is $z=40.4 \mathrm{~mm}$.

For the purpose of comparison a numerical characterization of realistic 3D model of ENZ lens is done by means of the commercial software CST Microwave Studio ${ }^{\mathrm{TM}}$ using the transient solver. The whole 3D model is built according to fabrication dimensions, including the bases on the top and bottom but excluding the crosspieces. Simulations results for transmission enhancement spectra along the optical z-axis are presented in the left panel of Fig. 2 (b). It can be noticed a slight frequency shift in the response, which is attributed to fabrication tolerances. At operational frequency the focal length is $38.91 \mathrm{~mm}$, which is in good agreement with experimental results.
Next, the $x y$-plane scanning was done at the position of the focal length $\mathrm{z}=40.4 \mathrm{~mm}$. The experimental normalized transmission enhancement is shown in Fig. 3 (b) where a clear focus can be observed. The measured maximum value of transmission enhancement is $5.61 \mathrm{~dB}$. The same results for simulations are shown in Fig. 3 (a). However, the maximum transmission enhancement is $16.77 \mathrm{~dB}$, which is significantly higher than in the experiment. This disagreement may be due to the non-uniform illumination of the lens in the experiment, resulting in the transmission reduction [18].

The experimental and simulation results of the transmission along $x$ - and $y$-axes at the each focal plane are plotted in Fig. 3 (c). Experimentally, the full width at half maximum (FWHM) is $2.21 \lambda_{0}$ and $1.69 \lambda_{0}$ for $x$ - and $y$-plane respectively. For simulations FWHM is $0.78 \lambda_{0}$ and $0.79 \lambda_{0}$ for $x$ - and $y$-plane respectively. It must be highlighted that the secondary lobes along the $x$-axis are higher than those along the $y$-axis. This is related with the effective aperture because, as described in the design section, the waveguide array is shorter along the $x$-axis causing the effective length to be $17 \%$ lower than the $y$-axis $\left(d_{y}\right.$ $>d_{x}$ ), and therefore, the energy focusing is worse along $x$. The value of depth of focus (DoF) is $14.8 \mathrm{~mm}=7.11 \lambda_{0}$ obtained in the experiment. In the simulation DoF is $3.94 \mathrm{~mm}=1.9 \lambda_{0}$. For ease of comparison all values are presented in Table 1.

(a)

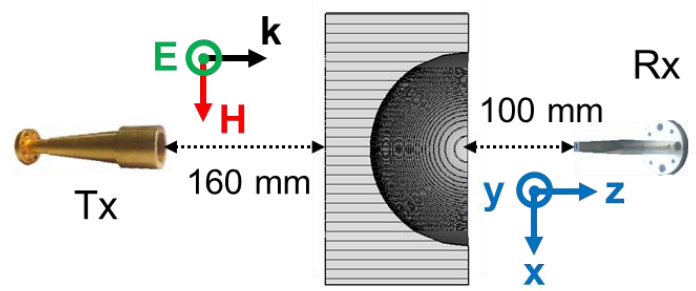

(b)
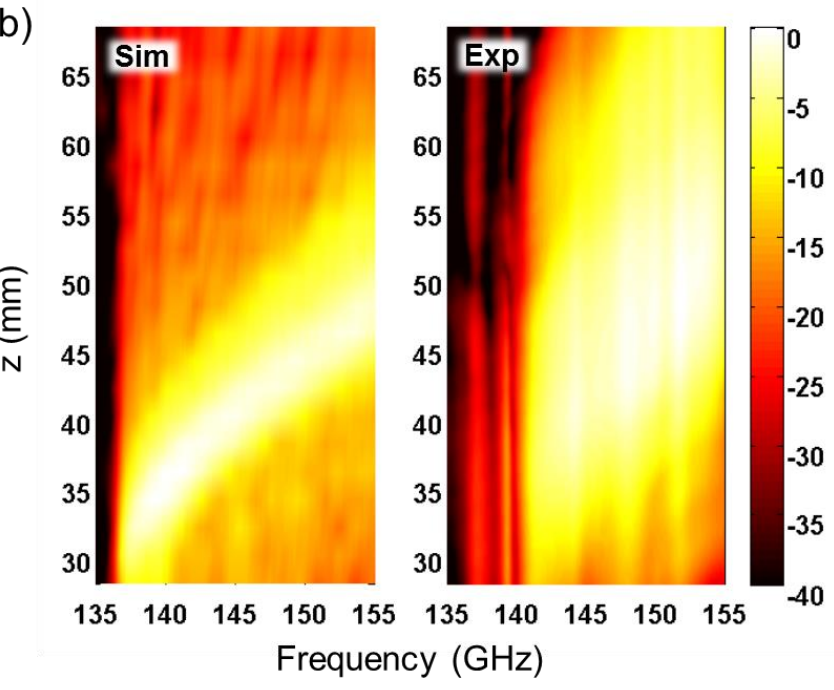

Fig. 2. (a) Sketch of the experimental set-up. (b) Normalized transmission enhancement spectra along the optical $z$-axis: (left) simulation, (right) measurement.

\section{B. Radiation characteristics}

To complete our study, the analysis of radiation properties was performed. A sketch of the experimental setup is shown in Fig. 4 (a). A waveguide probe WR-5.1 was placed at the experimental focus and used as a source while a waveguide 
probe WR-8.0 was used as a detector. A raster scanning was done in a rectangle of $200 \mathrm{~mm} \times 200 \mathrm{~mm}$ with a step of 0.8 $\mathrm{mm}$ at $100 \mathrm{~mm}$ away from the exit face of the lens.
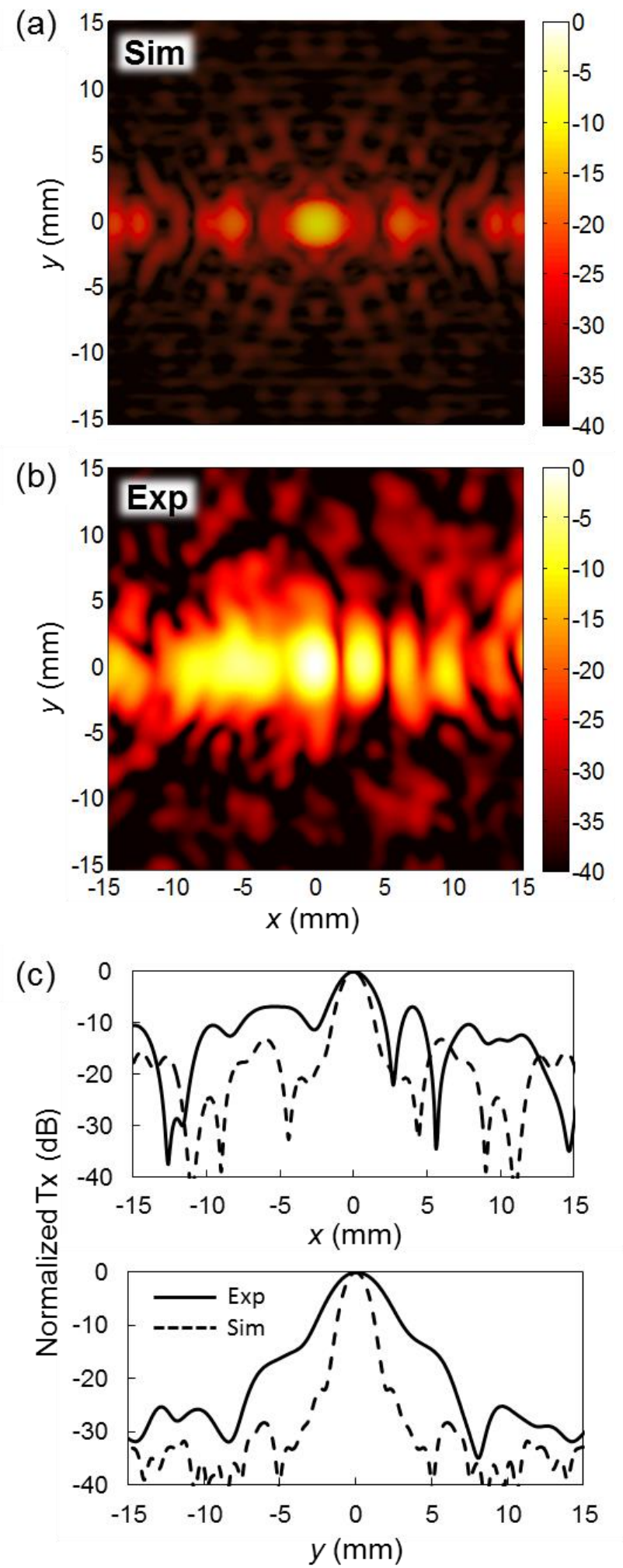

Fig. 3. (a) Simulated and (b) measured on the $x y$-plane normalized transmission enhancement at the working frequency $f_{0}=144 \mathrm{GHz}$. (c) Normalized transmission at $f_{0}$ along the $x$-axis (top) and along the $y$-axis (bottom) at the FL.
TABLE I

SUMMARY OF THE FOCUS PERFORMANCE

\begin{tabular}{|c|c|c|c|c|c|}
\hline \multirow{2}{*}{} & \multirow{2}{*}{$\mathbf{F L}^{\mathrm{a}}$} & $\mathbf{D o F}^{\mathrm{b}}$ & \multicolumn{2}{|c|}{ FWHM $^{\mathrm{c}}$} & \multirow{2}{*}{$\begin{array}{c}\text { Max. } \\
\text { Tx. }^{\mathrm{d}}\end{array}$} \\
\cline { 4 - 5 } & & & $x$-axis & $y$-axis & \\
\hline \multirow{3}{*}{ Exp } & $\begin{array}{c}40.40 \\
\mathrm{~mm}\end{array}$ & $\begin{array}{c}14.8 \mathrm{~mm} \\
7.11 \lambda_{0}\end{array}$ & $\begin{array}{c}2.36 \mathrm{~mm} \\
1.13 \lambda_{0}\end{array}$ & $\begin{array}{c}3.04 \mathrm{~mm} \\
1.46 \lambda_{0}\end{array}$ & $5.61 \mathrm{~dB}$ \\
\hline \multirow{2}{*}{ Sim } & $\begin{array}{c}38.91 \\
\mathrm{~mm}\end{array}$ & $\begin{array}{c}4.59 \mathrm{~mm} \\
2.21 \lambda_{0}\end{array}$ & $\begin{array}{c}1.63 \mathrm{~mm} \\
0.78 \lambda_{0}\end{array}$ & $\begin{array}{c}1.45 \mathrm{~mm} \\
0.69 \lambda_{0}\end{array}$ & $16.77 \mathrm{~dB}$ \\
\hline
\end{tabular}

${ }^{\mathrm{a}} \mathrm{FL}$ is the focal length

${ }^{b} \mathrm{DoF}$ is the depth of focus

${ }^{c} \mathrm{FWHM}$ is the full-width at half-maximum

${ }^{\mathrm{d}}$ Max. Tx. is the maximum transmission enhancemen

(a)

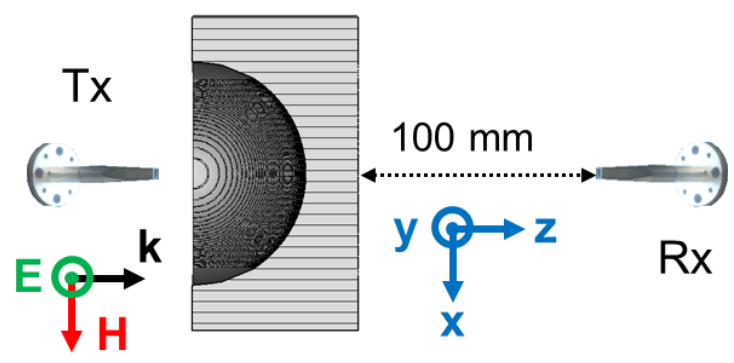

(b)

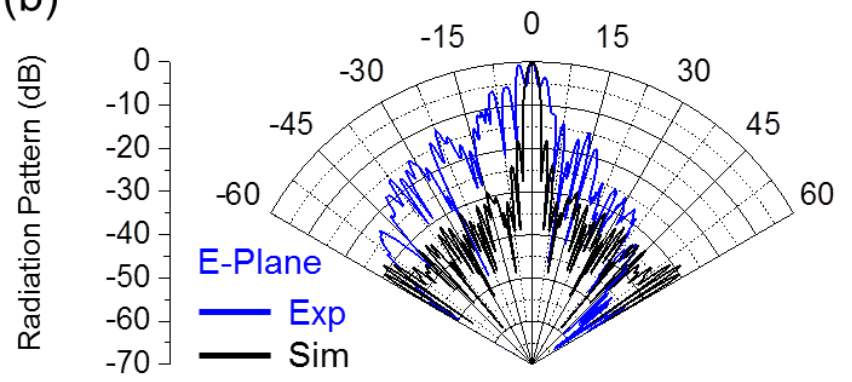

(c)

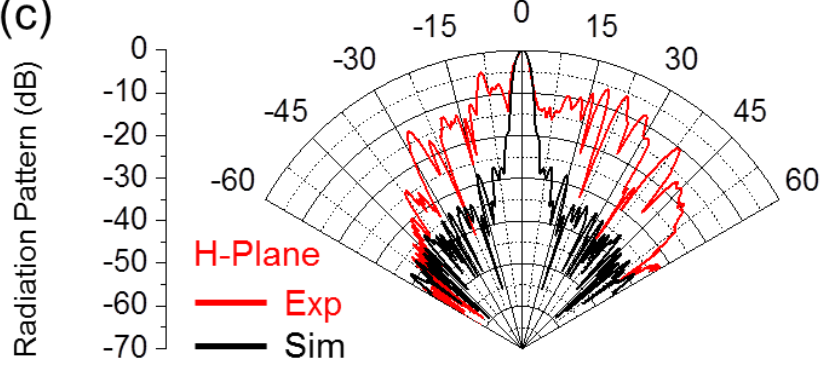

Fig. 4. (a) Sketch of the experimental set-up used for the far-field characterization. Measured and simulated co-polar radiation pattern on the Eplane (b) and H-plane (c).

To obtain the radiation pattern the planar near-field to farfield transformation was applied, which allows transforming the measured fields in this rectangle to the far-field region [17]. In the simulation only the end of the WR-8.0 is included and the radiation pattern is obtained by a far-field monitor. 
In Fig. $4(\mathrm{~b}, \mathrm{c})$ simulation and experimental results are shown for E- and H-plane, respectively. On the E-plane (Fig. 5(b)) experimental results demonstrate a narrow main lobe with a half-power beamwidth (HPBW) of $1.6 \mathrm{deg}$ and first null beam width (FNBW) equal to $2.8 \mathrm{deg}$. In the simulation a main lobe is obtained with a HPBW and FNBW of 2.2 deg and 5.6 $\mathrm{deg}$, respectively. In the experimental radiation pattern secondary lobes are higher than in the simulation that can be tied to undesired effect of the probe WR-5.1. In the experiment the first side-lobe levels (FSLL) are $-3.6 \mathrm{~dB}$ and $-0.8 \mathrm{~dB}$ while in the simulation FSLL is $-18.3 \mathrm{~dB}$ at both sides of the main lobe. On the H-plane (Fig. 5(c)) experimental results are presented with $\mathrm{HPBW}=3.0 \mathrm{deg}$ and $\mathrm{FNWB}=8.2 \mathrm{deg}$, which is in very good agreement with the simulated values of HPBW=2.9 deg and $F N W B=7.8 \mathrm{deg}$. The level of the secondary lobes is much lower: $-13.8 \mathrm{~dB}$ and $-7.8 \mathrm{~dB}$ for experimental results, and $-19.3 \mathrm{~dB}$ for the simulation. The measured directivity is equal to $17.6 \mathrm{dBi}$, and $25.4 \mathrm{dBi}$ in the simulation. All these results are presented in Table II.

TABLE II

RADIATION PATTERN PARAMETERS

\begin{tabular}{|c|c|c|c|c|c|c|c|}
\hline \multirow{5}{*}{} & \multicolumn{2}{|c|}{ HPBW $^{\mathrm{a}}$} & \multicolumn{2}{c|}{ FNBW $^{\mathrm{b}}$} & \multicolumn{2}{|c|}{ FSLL $^{\mathrm{c}}$} & \multirow{2}{*}{ Directivity } \\
\cline { 2 - 7 } & $\begin{array}{c}E- \\
\text { Plane }\end{array}$ & $\begin{array}{c}H- \\
\text { Plane }\end{array}$ & $\begin{array}{c}E- \\
\text { Plane }\end{array}$ & $\begin{array}{c}H- \\
\text { Plane }\end{array}$ & $\begin{array}{c}E- \\
\text { Plane }\end{array}$ & $\begin{array}{c}H- \\
\text { Plane }\end{array}$ & \\
\hline Exp & $\begin{array}{c}1.6 \\
\mathrm{deg}\end{array}$ & $\begin{array}{c}3.0 \\
\mathrm{deg}\end{array}$ & $\begin{array}{c}2.3 \\
\mathrm{deg}\end{array}$ & $\begin{array}{c}8.2 \\
\mathrm{deg}\end{array}$ & $\begin{array}{c}-3.6 \\
\mathrm{~dB}\end{array}$ & $\begin{array}{c}-13.8 \\
\mathrm{~dB}\end{array}$ & $17.6 \mathrm{dBi}$ \\
\hline Sim & $\begin{array}{c}2.2 \\
\mathrm{deg}\end{array}$ & $\begin{array}{c}2.9 \\
\mathrm{deg}\end{array}$ & $\begin{array}{c}5.6 \\
\mathrm{deg}\end{array}$ & $\begin{array}{c}7.8 \\
\mathrm{deg}\end{array}$ & $\begin{array}{c}-18.3 \\
\mathrm{~dB}\end{array}$ & $\begin{array}{c}-19.3 \\
\mathrm{~dB}\end{array}$ & $25.4 \mathrm{dBi}$ \\
\hline
\end{tabular}

${ }^{\text {a }} \mathrm{HPBW}$ is the half-power beamwidth

${ }^{\mathrm{b}} \mathrm{FNBW}$ is the first null beam width

${ }^{\mathrm{c}} \mathrm{FSLL}$ is the first side-lobe level

\section{CONCLUSIONS}

In this work, an ENZ lens with a plano-concave profile has been designed, fabricated and demonstrated experimentally. The design consist of a 2D array of narrow hollow rectangular waveguides working near the cutoff frequency $f_{0}=144 \mathrm{GHz}$ of the fundamental mode $\mathrm{TE}_{10}$. Experimental results show a good agreement with simulation results. A focal length found at $\mathrm{z}=$ $40.4 \mathrm{~mm}$ and $\mathrm{z}=38.91 \mathrm{~mm}$ for experimental and simulation, respectively. The effective permittivity of the lens at the operational frequency in the experiment $144 \mathrm{GHz}$ is 0.103 , demonstrating that the lens mimics a medium with permittivity near zero. For the working frequency the measured directivity is equal to $17.6 \mathrm{dBi}$. The ENZ lens presented in this work demonstrates possibilities of using waveguide-based ENZ metamaterials in applications such as graded-index lenses, prisms or beam steerers.

\section{ACKNOWLEDGMENT}

This work was supported in part by the Spanish Government under contract Consolider Engineering Metamaterials CSD2008-00066 and contract TEC2011-28664C02-01. B. O. is sponsored by Spanish Ministerio de Economía y Competitividad under grant FPI BES-2012-054909. V.T. is sponsored by the Universidad Pública de Navarra. V.P.-P. is sponsored by Spanish Ministerio de Educación, Cultura y Deporte under grant FPU AP-2012-3796. M.B. is sponsored by the Spanish Government via RYC-2011-08221. M. N.-C. is supported by the Imperial College Junior Research Fellowship. In memoriam of Prof. Mario Sorolla.

\section{REFERENCES}

[1] R. A. Shelby, D. R. Smith, and S. Schultz, "Experimental verification of a negative index of refraction.," Science, vol. 292, no. 5514, pp. 77-9, Apr. 2001.

[2] V. M. Shalaev, "Optical negative-index metamaterials," Nat. Photonics, vol. 1, no. 1, pp. 41-48, Jan. 2007.

[3] Z. Liu, H. Lee, Y. Xiong, C. Sun, and X. Zhang, "Far-field optical hyperlens magnifying sub-diffraction-limited objects.," Science, vol. 315, no. 5819, p. 1686, Mar. 2007.

[4] M. Silveirinha, A. Alù, and N. Engheta, "Parallel-plate metamaterials for cloaking structures," Phys. Rev. E, vol. 75, no. 3 , p. 036603, Mar. 2007.

[5] A. Silva, F. Monticone, G. Castaldi, V. Galdi, A. Alù, and N. Engheta, "Performing mathematical operations with metamaterials.," Science, vol. 343, no. 6167, pp. 160-3, Jan. 2014.

[6] A. Demetriadou and Y. Hao, "Slim Luneburg lens for antenna applications.," Opt. Express, vol. 19, no. 21, pp. 19925-34, Oct. 2011.

[7] M. Silveirinha and N. Engheta, "Tunneling of Electromagnetic Energy through Subwavelength Channels and Bends using $\varepsilon$-NearZero Materials," Phys. Rev. Lett., vol. 97, no. 15, p. 157403, Oct. 2006.

[8] A. Alù, M. G. Silveirinha, A. Salandrino, and N. Engheta, "Epsilonnear-zero metamaterials and electromagnetic sources : Tailoring the radiation phase pattern," pp. 1-13, 2007.

[9] B. Edwards, A. Alù, M. Silveirinha, and N. Engheta, "Experimental Verification of Plasmonic Cloaking at Microwave Frequencies with Metamaterials," Phys. Rev. Lett., vol. 103, no. 15, p. 153901, Oct. 2009

[10] A. Monti, F. Bilotti, A. Toscano, and L. Vegni, "Possible implementation of epsilon-near-zero metamaterials working at optical frequencies," Opt. Commun., vol. 285, no. 16, pp. $3412-$ 3418, Jul. 2012.

[11] C. Argyropoulos, P.-Y. Chen, G. D’Aguanno, N. Engheta, and A. Alù, "Boosting optical nonlinearities in $\varepsilon$-near-zero plasmonic channels," Phys. Rev. B, vol. 85, no. 4, p. 045129, Jan. 2012.

[12] N. Engheta, "Circuits with light at nanoscales: optical nanocircuits inspired by metamaterials.," Science, vol. 317 , no. 5845 , pp. 1698 702, Sep. 2007

[13] V. Torres, B. Orazbayev, V. Pacheco-Peña, J. Teniente, M. Beruete, M. Navarro-Cía, M. Sorolla, and N. Engheta, "Experimental Demonstration of a Millimeter-Wave Metallic ENZ Lens based on the Energy Squeezing Principle," submitted.

[14] M. Silveirinha and N. Engheta, "Theory of supercoupling, squeezing wave energy, and field confinement in narrow channels and tight bends using $\varepsilon$ near-zero metamaterials," Phys. Rev. B, vol. 76, no. 24, p. 245109, Dec. 2007.

[15] A. Alù, M. Silveirinha, A. Salandrino, and N. Engheta, "Epsilonnear-zero metamaterials and electromagnetic sources: Tailoring the radiation phase pattern," Phys. Rev. B, vol. 75, no. 15, p. 155410 , Apr. 2007.

[16] M. Navarro-Cía, M. Beruete, M. Sorolla, and N. Engheta, "Lensing system and Fourier transformation using epsilon-near-zero metamaterials," Phys. Rev. B, vol. 86, no. 16, p. 165130, Oct. 2012.

[17] S. Gregson, J. McCormick, and C. Parini, Principles of Planar Near-Field Antenna Measurements. 2007.

[18] V. Torres, V. Pacheco-Peña, P. Rodríguez-Ulibarri, M. NavarroCía, M. Beruete, M. Sorolla, and N. Engheta, "Terahertz epsilonnear-zero graded-index lens.," Opt. Express, vol. 21, no. 7, pp. 9156-66, Apr. 2013. 